\title{
Clinical Outcome of Patients with Acute Coronary Syndrome (ACS) Admitted at Coronary Care Unit (CCU) at Benha University Hospital: Gender Specific Differences \\ M.K.Mahmoud, M.A.Hamouda and M.M.Ali \\ Cardiology Dept., Faculty of Medicine, Benha Univ., Benha, Egypt \\ E-Mail: Marwa268@Gmail.com
}

\begin{abstract}
Cardiovascular disease (CVD) remains the most widely recognized reason for bleakness and mortality in Europe, representing $49 \%$ of passings in ladies and $40 \%$ of passings in men [1]. Over the most recent forty years, ageadjusted mortality for CVD has ceaselessly declined, notwithstanding, less significantly in ladies than in men [2]. Most intriguingly, ongoing investigations report a critical expansion in the event that casualty paces of intense coronary conditions (ACS) in young ladies $<55$ years old, while a reduction in mortality fromcoronary corridor sickness (CAD) has happened in more youthful men [3]. Despite developing proof exhibiting sex and sex contrasts in standard danger factors, coronary life systems and capacity, manifestations introduction, comorbidities, treatment viability, and results of ACS, instruments behind these distinctions are to a great extent unexplored [4]. These information holes are sustained by the persevering underrepresentation of ladies in cardiovascular clinical preliminaries and an absence of essential science proof acquired from female creatures and cells attributable to a complex disproved worry that incorporation of females will build changeability, just as twofold example size and expenses [5]. This survey gives an outline of contemporary proof revealing insight into sex and sexual orientation contrasts in the clinical introduction of ACS just as in symptomatic precision of tests, obtrusive treatment, pharmacotherapy, and outcomes.
\end{abstract}

Keywords: Acute Coronary Syndrome, Gendre Specific differences.

\section{Introduction}

Increasing proof backings the thought that obstructive CAD alone is lacking to clarify manifestations of myocardial ischaemia [6]. Indeed, microvascular angina, characterized as indications and target proof of myocardial ischemia alongside non-obstructive CAD ( $<50 \%$ coronary distance across decrease or potentially fragmentary stream save >0.80) and a decrease of CFR and additionally inducible microvascular fit, [7] influences around half of patients with non-obstructive $\mathrm{CAD}$ or ordinary imaging findings.8Despite the nonappearance of obstructive epicardial stenosis in these patients, they may give extreme myocardial (ischaemia and non-obstructive CAD) or even (MI and non-obstructive CAD). These conditions are all the more regularly saw in ladies, given the lower weight of obstructive

Computer aided design in the female populace Fig (2). Truth be told, the presence of microvascular angina is twice as pervasive in ladies than men [8]. The previous predicts an especially high danger in ladies, as occasion free endurance is diminished in ladies with weakened CFR or coronary reactivity [9]. As PET-determined CFR mirrors the haemodynamic impacts of central, diffuse and microvascular $\mathrm{CAD}$ on myocardial tissue perfusion, hindered CFR is by all accounts a significant objective for coronary microvascular illness (CMVD) hazard decrease in ladies. Without a doubt, CMVD may likewise add to the pathogenesis of $\mathrm{HFpEF}$, another condition all the more usually saw in ladies. 10 Thus, is it pivotal that indicative patients who don't show provincial ischaemia related with stream restricting epicardial CAD go through additional testing.

Subsequently, this examination is intended To assess the clinical result of patients with intense coronary condition

(ACS) with respect to sex explicit differences .

\section{Patients and methods}

2.1 Study Design
This will be an observational ,case series ,single center study that will include all patients with acute coronary syndrome(ACS) who will be admitted at coronary care unit at "Benha University hospital" in the period from April 2019 to April 2020.

\subsection{Study protocol}

- This study will evaluate clinical outcome of this category of patients both inhospital stay and at 6 months follow up .

- In addition to left ventricular remodeling in patients with STEMI using speckle tracking (GLPSS) will be done at baseline and 6 months follow up .

- The study will primarily compare the outcomes between males and females patients with ACS.

\subsection{Primary end point}

6 months mortality ,myocardial infarction ,stroke,revasularization .

\subsection{Secondary end point}

6 months GLPSS in patients with STEMI .

\section{Methods}

The included patients will be subjected to the following:

\section{Benchmark assessment}

All patients will have survey of clinical history including

Age, sex, Risk Factors of coronary supply route sickness (DM-HTN-Dyslipidemia-smoking), earlier history of coronary conduit infection, ,earlier history of mediation, other comorbidities, drugs.

\section{Full clinical assessment}

With specific accentuation on the beat and circulatory strain of the patients, just as auscultation of the back to evoke the presence of any clinically distinguishable aspiratory venous clog, auscultation of the heart for the presence of third heart sounds or discernible mumbles 


\section{Benchmark Electrocardiography}

Twelve leads ECG will be accomplished for every patient

Heart biomarkers: including troponins and CKMB

Benchmark Echocardigraphy

All patients will be assessed by echocardiography for the appraisal of provincial divider irregularities and generally speaking left ventricular systolic capacity .

Follow up

- 6 months follow up will incorporate appraisal of clinical factors among guys and females patients including all reason mortality,myocardial infarction, stroke, heart disappointment, revascularization

- Sub gathering investigation of patients with STEMI will incorporate a half year left ventricular renovating utilizing dot following (GLPSS)

Spot following echocardiography

Longitudinal strain estimations with the execution of 2D dot following will be proceeded as recently portrayed 11 End-systole will be recognized as relating to the aortic valve conclusion estimated by beat Doppler. The administrator will follow the endocardial verge on an enddiastolic edge and the product consequently followed the fringe on the resulting outlines. Satisfactory following would then be able to be confirmed continuously and revised whenever considered significant by changing the area of interest or physically remedying the outskirt to guarantee ideal following. After the following cycle is finished myocardial disfigurement is plotted in time versus strain charts, where it is conceivable to recognize the various periods of cardiovascular cycle.

Circumferential, spiral strain boundaries and their progressions from rest (BASE) to low pressure (MIN), top pressure (MAX) and recuperation (REC), will be assessed. Pinnacle spiral and circumferential strain (systolic and early diastolic) will be estimated from mid short-hub see very still, low and high dobutamine dosages. Pinnacle longitudinal strain will be estimated from apical 4-, 3chamber and 2-chamber sees. Worldwide strain examination will be performed. As per the current rules, GLS will be determined from circles procured from 2-, 3and 4-chamber sees 11 .

\section{Results and conversation}

This examination was done on all patients with intense coronary disorder (ACS) admitted to the coronary consideration unit at "Benha University clinic" from April 2019 to April 2020.

General qualities in the entire investigation populace

The mean age of the examination populace was 58 years, with a standard deviation of 12 years. As respect sex, $66.0 \%$ were guys, while $34.0 \%$ were females. $39.8 \%$ and $46.7 \%$ were diabetic and hypertensive, individually. $41.2 \%$ were smokers. $41.2 \%$ had a background marked by ischemia; of them, $26.3 \%$ did PCI, $10.6 \%$ did CABG. Comorbidities were found in $14.5 \%$, and dyslipidemia was found in

\section{$3.1 \%$ of the investigation population}

Table (1) General characteristics in the whole study population.

\begin{tabular}{|c|c|c|c|}
\hline \multicolumn{4}{|c|}{ General characteristics } \\
\hline Age (years) & \multicolumn{2}{|c|}{ Mean \pm SD } & $58 \pm 12$ \\
\hline \multirow[t]{2}{*}{ Gender } & Males & $\mathrm{n}(\%)$ & $678(66.0)$ \\
\hline & Females & $\mathrm{n}(\%)$ & $350(34.0)$ \\
\hline Diabetes mellitus & Yes & $\mathrm{n}(\%)$ & $409(39.8)$ \\
\hline Hypertension & Yes & $\mathrm{n}(\%)$ & $480(46.7)$ \\
\hline Smoking & Yes & $\mathrm{n}(\%)$ & $424(41.2)$ \\
\hline H/O ischemia & Yes & $\mathrm{n}(\%)$ & $255(24.8)$ \\
\hline H/O PCI* & Yes & $\mathrm{n}(\%)$ & $67(26.3)$ \\
\hline H/O CABG ${ }^{*}$ & Yes & $\mathrm{n}(\%)$ & $27(10.6)$ \\
\hline Comorbidities & Yes & $\mathrm{n}(\%)$ & $149(14.5)$ \\
\hline Dyslipidemia & Yes & $\mathrm{n}(\%)$ & $32(3.1)$ \\
\hline
\end{tabular}

*Percentage was calculated based on total 255 had ischemia.

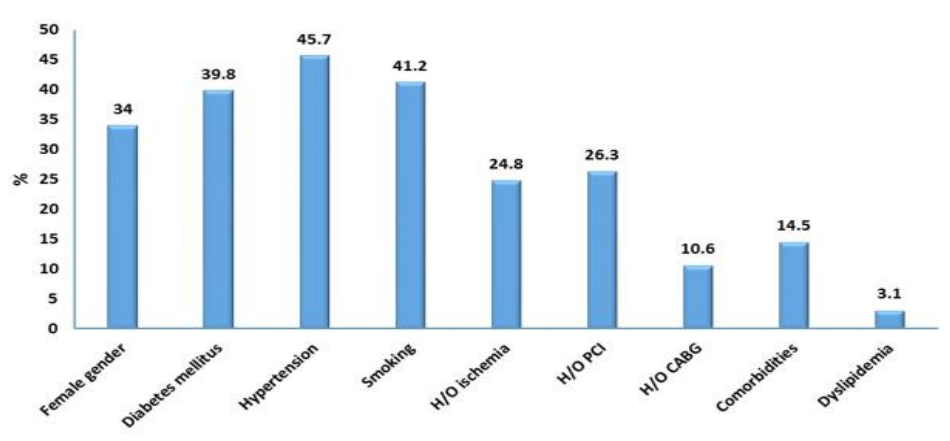

Fig (4) General characteristics in the whole study population.

\section{Clinical examination findings in the whole study population}

The mean heart rate was $80 \mathrm{~b} / \mathrm{m}$, with a standard deviation of $19 \mathrm{~b} / \mathrm{m}$. Mean systolic and diastolic blood pressure were $127 \& 80$, respectively. $2.0 \%$ were shocked. As regard Killip class, the majority $(89.3 \%)$ were class I. 


\begin{tabular}{lcc}
\hline Clinical signs & & \\
\hline Heart rate & Mean \pm SD & $80 \pm 19$ \\
Systolic blood pressure & Mean \pm SD & $127 \pm 25$ \\
Diastolic blood pressure & Mean \pm SD & $80 \pm 14$ \\
Shock & Yes $\mathrm{n}(\%)$ & $21(2.0)$ \\
KILLIP CLASS & I $\mathrm{n}(\%)$ & $918(89.3)$ \\
& II $\mathrm{n}(\%)$ & $76(7.4)$ \\
& III $\mathrm{n}(\%)$ & $27(2.6)$ \\
\hline
\end{tabular}

\section{Presenting symptoms in the whole study population}

$95.3 \%$ presented with chest pain. $4.7 \%$ presented with dyspnea, and $1.9 \%$ presented with arrhythmia

Table (3) Presenting symptoms in the whole study population.

\begin{tabular}{lr}
\hline & N (\%) \\
\hline Chest pain & $980(95.3)$ \\
Dyspnea & $48(4.7)$ \\
Arrhythmia & $20(1.9)$ \\
\hline
\end{tabular}

Clinical presentation in the whole study population

The majority of the study population presented with STEM (48.3\%), 16.0\% were NSTEMI, and 35.0\% presented with unstable angina.

Table (4) Clinical presentation in the whole study population.

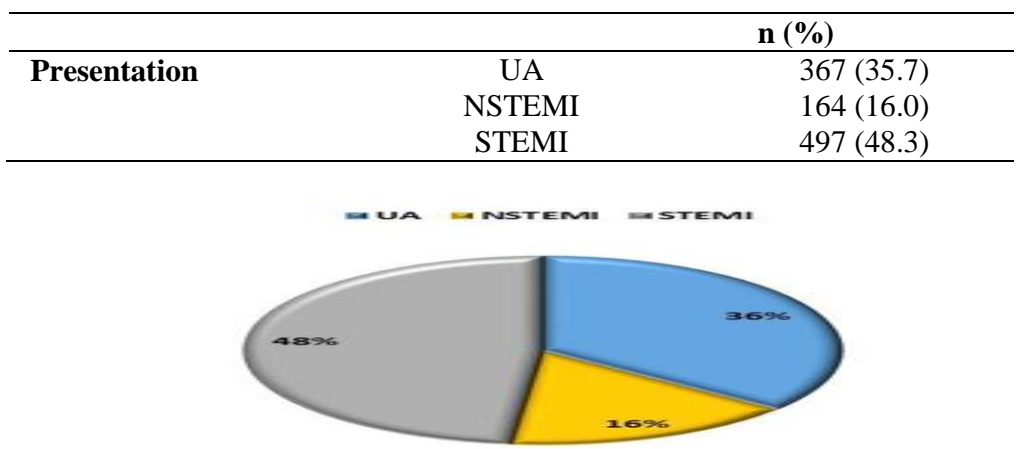

Fig (5) Distribution of clinical presentation in the whole study population.

\section{Vessels affected in those with STEMI}

The most frequent vessel affected was anterior (24.7\%) followed by inferior $(13.9 \%)$, while the least frequent vessels involved were posterior and inferolateral ( $1 \%$ and $0.6 \%$, respectively)

Table (10) Vessels affected in those with STEMI.

\begin{tabular}{lc}
\hline & $\mathbf{N}(\%)$ \\
\hline Anterior & $123(24.7)$ \\
Anteroseptal & $20(4.0)$ \\
Extensive anterior & $31(6.2)$ \\
High lateral & $26(5.2)$ \\
Missed anterior & $49(9.9)$ \\
Inferior & $69(13.9)$ \\
Inferolateral & $3(0.6)$ \\
INF RT & $40(8.0)$ \\
INF post & $40(8.0)$ \\
INF RT post & $27(5.4)$ \\
INF RT LAT post & $21(4.2)$ \\
Posterior & $5(1.0)$ \\
Missed inf & $25(5.0)$ \\
Anterolateral & $17(3.4)$ \\
Posterolateral & $7(1.4)$ \\
\hline
\end{tabular}

All percentages were calculated based on a total of 497 patients with STEMI 


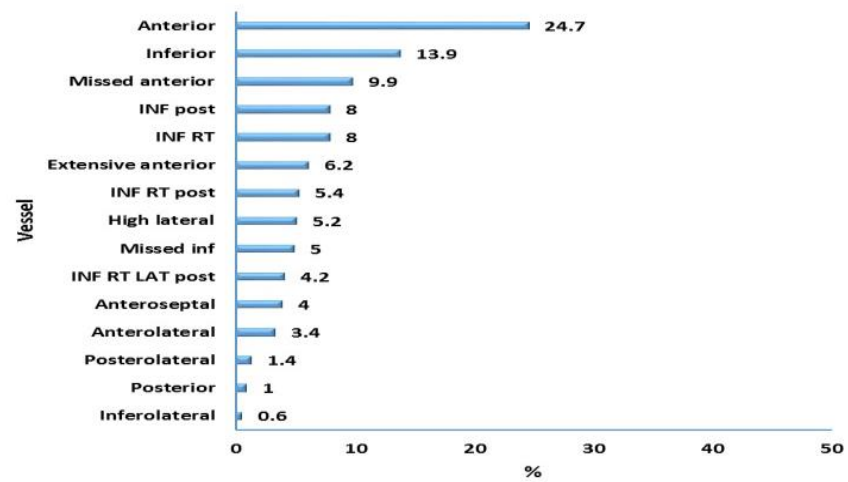

Fig (6) Vessels affected in those with STEMI.

Echocardiography findings in the whole study population

Mean EF was 54 with a standard deviation of 12. RWMA was present in $(65.9 \%)$ of the study population. Mild mitral regurge was found in $42.5 \%$ of the study

population, moderate and severe mitral regurge represented $11.7 \%$ and $6,8 \%$, respectively. VSR was found in only one patient. Mean EDD and ESD were 4.88 and 3,73, respectively.

3D EF was 50 with a standard deviation of 12 .

Table (11) Echocardiographic findings in the whole study population.

\begin{tabular}{lccr}
\hline Echocardiography & & & \\
\hline EF & Mean \pm SD & & $54 \pm 12$ \\
RWMA & Yes n $(\%)$ & & $677(65.9)$ \\
MR & No MR & $\mathrm{n}(\%)$ & $401(39.0)$ \\
& Mild & $\mathrm{n}(\%)$ & $437(42.5)$ \\
& Moderate & $\mathrm{n}(\%)$ & $120(11.7)$ \\
VSR & Severe & $\mathrm{n}(\%)$ & $70(6.8)$ \\
EDD & Yes n $(\%)$ & & $1(0.1)$ \\
ESD & Mean \pm SD & & $4.88 \pm 1.04$ \\
3D EF & Mean \pm SD & & $3.73 \pm 0.91$ \\
\end{tabular}
$\mathrm{EF}=$ Ejection fraction
RWMA = Right wall motion abnormality
MR = Mitral regurge
$\mathrm{VSR}=$ Ventricular septal rupture $\mathrm{EDD}=$ End diastolic dimension
$\mathrm{ESV}=$ End systolic dimension

\section{GLPSS baseline \& at 6months in the whole study population}

Mean GLPSS at baseline was -16.9 with a standard deviation of 3.6. At 6months, the Mean GLPSS was -13.1 with a standard deviation of 3.1. P-value was $<0.001$

Table (12) GLPSS at baseline \& 6 months.

\begin{tabular}{lcccc}
\hline & & $\begin{array}{c}\text { Males } \\
(\mathbf{n = 6 7 8})\end{array}$ & $\begin{array}{c}\text { Females } \\
(\mathbf{n = 3 5 0})\end{array}$ & P value \\
\hline Baseline & Mean \pm SD & $-16.7 \pm 3.7$ & $-17.3 \pm 3.5$ & 0.012 \\
At six months & Mean \pm SD & $-13.2 \pm 3.1$ & $-12.8 \pm 2.9$ & .052 \\
& & & & \\
\%change & Median (range) & $-22.28(-58.22-130.77)$ & $-30.44(-56.37-122.47)$ & 0.002 \\
\hline
\end{tabular}

Paired t-test was used GLPSS $=$ Global Longitudinal Peak Systolic Strain

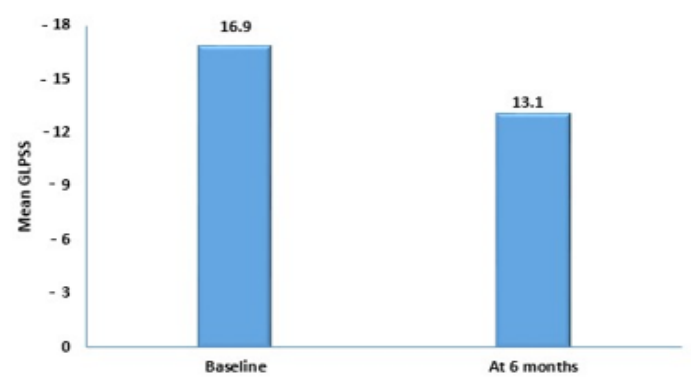

Fig (7) GLPSS at baseline \& 6 months. 
The most frequent complication was mortality $(4.7 \%)$, followed by re-hospitalization (4.2\%), re-infarction (1.2\%), stroke $(1.1 \%)$ and hemorrhage $(0.8 \%)$.

Table (13) Complications in the whole study population.

\begin{tabular}{lr}
\hline & $\mathbf{N}(\%)$ \\
\hline Re-infarction & $12(1.2)$ \\
Stroke & $11(1.1)$ \\
Hemorrhage & $7(0.8)$ \\
Re-hospitalization & $53(4.2)$ \\
Mortality & $48(4.7)$ \\
\hline
\end{tabular}

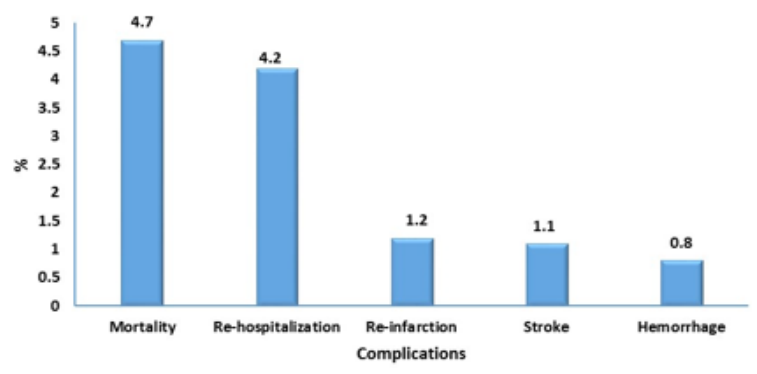

Fig (8) Complications in the whole study population.

Six months follow up in the whole study population

Re-ischemia occurred in $12.9 \%$ of the study population. Revascularization occurred in $6.0 \%$. Heart failure occurred in $5.1 \%$. Mortality occurred in $1.0 \%$, and stroke occurred in $0.3 \%$

Table (14) Follow up findings in the whole study population.

\begin{tabular}{lc}
\hline & $\mathbf{N}(\%)$ \\
\hline Re-ischemia & $133(12.9)$ \\
Stroke & $3(0.3)$ \\
Heart failure & $52(5.1)$ \\
Re-vascularization & $62(6.0)$ \\
Mortality & $10(1.0)$ \\
\hline
\end{tabular}

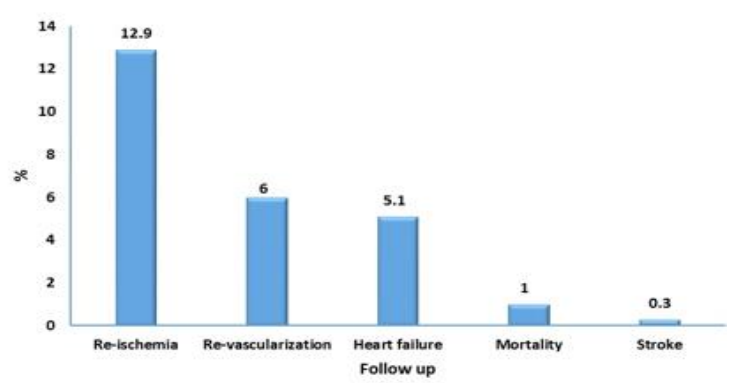

Fig (9) Follow up findings in the whole study population

Management lines in the whole study population

$85.9 \%$ of patients received conservative management, $32.5 \%$ received thrombolytic therapy, and $5.8 \%$ underwent intervention.

Table (15 ) Lines of management in the whole study population.

\begin{tabular}{lc}
\hline & $\mathbf{N}(\boldsymbol{\%})$ \\
\hline Conservative & $605(58.9)$ \\
Thrombolytic & $334(32.5)$ \\
Intervention & $60(5.8)$ \\
\hline
\end{tabular}

\section{Type of intervention}

PPCI was the most frequent intervention, followed by rescue PCI (25.7\%) and emergency PCI (21.7\%). The least frequent interventions were GP (6.7\%) and pharmaco-invasive PCI (1.7\%).

Table (16 ) Type of intervention in those received interventional therapy. 


\begin{tabular}{lc}
\hline P PTCA & $8(13.3)$ \\
Emergency PCI & $13(21.7)$ \\
Table (16) Continue & \\
\hline Rescue PCI & $15(25.7)$ \\
GP & $4(6.7)$ \\
Pharmaco-invasive PCI & $1(1.7)$ \\
\hline
\end{tabular}

Percentages were calculated based on a total of 60 patients did intervention PPCI = Primary Percutaneous Coronary Intervention PTC $=$ Percutaneous transluminal coronary angioplasty

\section{Discussion}

Our examination indicated that, The mean age of the investigation populace was 58 years, with a standard deviation of 12 years. As respect sex, $66.0 \%$ were guys, while $34.0 \%$ were females. $39.8 \%$ and $46.7 \%$ were diabetic and hypertensive, individually. $41.2 \%$ were smokers. $41.2 \%$ had a background marked by ischemia; of them, $26.3 \%$ did PCI, $10.6 \%$ did CABG. Comorbidities were found in $14.5 \%$, and dyslipidemia was found in $3.1 \%$ of the examination populace. what's more, those information with Data arising out of the VIRGO study show that youthful and moderately aged ladies hospitalized for type 1 and type $2 \mathrm{MI}$ were bound to have lower financial status, more significant levels of psychosocial trouble, for example, melancholy and more unfortunate physical/emotional well-being, and by and large lower personal satisfaction contrasted with men [12].

Albeit customary danger factors for CVD are the equivalent in ladies and men, contrasts in pervasiveness and effect of these danger factors fluctuate between the genders [13]. Also, this was relating to our examination results .

This is particularly found in ACS, as ladies who present with ACS are by and large more established and have more comorbidities, including a higher predominance of hypertension, dyslipidaemia, diabetes, cardiovascular breakdown, and atrial fibrillation [14].

The dangers of apoplexy and draining contrast among people. These sex contrasts have been ascribed to the higher time of ladies with ACS, comorbidities, and body weight [15]. Moreover, ladies experience variances of supportive of thrombotic action and haemostasis that are identified with the period, the utilization of hormonal contraceptives or HRT, pregnancy, and menopause [15].

All of which may add to sex contrasts in the thrombotic or haemorrhagic weight in ladies with ACS. To be sure, the higher danger of draining entanglements during PCI saw in ladies may to a limited extent be identified with an estrogen-subordinate increment of prostacyclin emission and nitric oxide bioavailability just as a direct inhibitory impact of estrogen on platelet aggregation [16].

\section{Conclusion}

Although These sex contrasts have been ascribed to the higher time of ladies with ACS, comorbidities, and body weight. 15 moreover, ladies experience variances of supportive of thrombotic action and haemostasis that are identified with the period, the utilization of hormonal contraceptives or HRT, pregnancy, and menopauseresult of a persistent underrepresentation of women in cardiovascular studies [17].

\section{References}

[1] N.Townsend, L.Wilson, P.Bhatnagar, K.Wickramasinghe, Cardiovascular disease in Europe: epidemiological update 2016. Eur Heart J, Vol.37, PP.3232-3245,2016.
[2] A.Gupta, Y .Wang, J.A.Spertus, M.Geda, Trends in acute myocardial infarction in young patients and differences by sex and race, 2001 to 2010. J Am Coll Cardiol, Vol.64, PP.337-345,2014.

[3] N.R.Smilowitz, N.Gupta, Y.Guo, J.Zhong, Acute myocardial infarction during pregnancy and the puerperium in the United States. Mayo Clin Proc, Vol.93, PP.1404-1414,2018.

[4] V.Vaccarino, Myocardial infarction in young women. Circulation, Vol.139, PP. 1057-1059,2019.

[5] Buch T, Moos K, Ferreira FM, Frohlich H, Gebhard $\mathrm{C}$, Tresch A. Benefits of a factorial design focusing on inclusion of female and male animals in one experiment. J Mol Med (Berl) 2019;97:871-877.

[6] C.Pizzi, B.Xhyheri, G.M.Costa, M.Faustino, Nonobstructive versus obstructive coronary artery disease in acute coronary syndrome: a meta-analysis. J Am Heart Assoc, Vol.5, PP. e004185,2016.

[7] P.Ong, P.G.Camici, J.F. Beltrame, F.Crea, International standardization of diagnostic criteria for microvascular angina. Int J Cardiol, Vol.250, PP.1620,2018 .

[8] L.Jespersen, A.Hvelplund, S.Z.Abildstrom, F.Pedersen, Stable angina pectoris with no obstructive coronary artery disease is associated with increased risks of major adverse cardiovascular events. Eur Heart J, Vol.33, PP.734-744,2012.

[9] V.R.Taqueti, L.J. Shaw, N.R.Cook, V.L.Murthy, Excess cardiovascular risk in women relative to men referred for coronary angiography is associated with severely impaired coronary flow reserve, not obstructive disease. Circulation, Vol.135, PP.566$577,2017$.

[10] K .Srivaratharajah, T.Coutinho, R. deKemp, P. Liu, Reduced myocardial flow in heart failure patients with preserved ejectionfraction. Circ Heart Fail, Vol.9, PP.e002562,2016.

[11] C.Aggeli, I.Felekos, D.Tousoulis, E.Gialafos, Myocardial mechanics for the early detection of cardiac sarcoidosis. International journal of cardiology, Vol. 168(5), PP. 4820-4821, 2013.

[12] E.M.Bucholz, K.M.Strait, R.P.Dreyer, S.T.Lindau, Editor's choice-sex differences in young patients with acute myocardial infarction: a VIRGO study analysis. Eur Heart J Acute Cardiovasc Care.6, PP.610622,2017

[13] M.Garcia, S.L.Mulvagh, C.N.Merz, J.E. Buring, Cardiovascular disease in women: clinical perspectives. Circ Res, Vol.118, PP.1273-1293,2016.

[14] J.A. Udell, M.Koh, F.Qiu, P.C.Austin, Outcomes of women and men with acute coronary syndrome treated with and without percutaneous coronary revascularization. J Am Heart Assoc, Vol.6, PP.e004319,2017.

[15] G.Renda, G.Patti, I.M .Lang, J.M.Siller-Matula, Working Group on Thrombosis of the Italian Society of Cardiology. Thrombotic and hemorrhagic burden in women: gender-related issues in the response to 
antithrombotic therapies. Int J Cardiol.286, PP.198207,2019.

[16] M.R.Sowers, K.A.Matthews, M .Jannausch, J.F. Randolph, Hemostatic factors and estrogen during the menopausal transition. J Clin Endocrinol Metab, Vol.90, PP.5942-5948,2005.
[17] P.E.Scott, E.F.Unger, M.R.Jenkins, M.R.Southworth, Participation of women in clinical trials supporting FDA approval of cardiovascular drugs. J Am Coll Cardiol, Vol.71, PP.19601969,2018. 